\title{
A Simple Java Code Generator for ACL2 Based on a Deep Embedding of ACL2 in Java
}

\author{
Alessandro Coglio \\ Kestrel Institute \\ http://www.kestrel.edu
}

\begin{abstract}
AIJ (ACL2 In Java) is a deep embedding in Java of an executable, side-effect-free, non-stobjaccessing subset of the ACL2 language without guards. ATJ (ACL2 To Java) is a simple Java code generator that turns ACL2 functions into AIJ representations that are evaluated by the AIJ interpreter. AIJ and ATJ enable possibly verified ACL2 code to run as, and interoperate with, Java code, without much of the ACL2 framework or any of the Lisp runtime. The current speed of the resulting Java code may be adequate to some applications.
\end{abstract}

\section{Motivation and Contributions}

A benefit of writing code in a theorem prover is the ability to prove properties about it, such as the satisfaction of requirements specifications. A facility to generate code in one or more programming languages from an executable subset of the prover's logical language enables the possibly verified code to run as, and interoperate with, code written in those programming languages. Assuming the correctness of code generation (whose verification is a separable problem, akin to compilation verification) the properties proved about the original code carry over to the generated code.

The ACL2 theorem provers's tight integration with the underlying Lisp platform enables the executable subset of the ACL2 logical language to run readily and efficiently as Lisp, without the need for explicit code generation facilities. Nonetheless, some situations may call for running ACL2 code in other programming languages: specifically, when the ACL2 code must interoperate with external code in those programming languages in a more integrated and efficient way than afforded by inter-language communication via foreign function interfaces [4, 9], or by inter-process communication with the ACL2/Lisp runtime via mechanisms like the ACL2 Bridge [2, : doc bridge]. Using Lisp implementations written in the target programming languages [1] involves not only porting ACL2 to them, but also including much more runtime code than necessary for the target applications. Compilers from Lisp to the target programming languages may need changes or wrappers, because executable ACL2 is not quite a subset of Lisp; furthermore, the ability to compile non-ACL2 Lisp code is an unnecessary complication as far as ACL2 compilation is concerned, making potential verification harder.

The work described in this paper contributes to the goal of running ACL2 code in other programming languages in the integrated manner described above:

- ATJ (ACL2 To Java) is a Java code generator for ACL2. ATJ translates executable, side-effectfree, non-stobj-accessing ACL2 functions, without their guards, into Java. It does so in a simple way, by turning the functions into deeply embedded Java representations that are executed by an ACL2 evaluator written in Java.

- AIJ (ACL2 In Java) is a deep embedding in Java of an executable, side-effect-free, non-stobjaccessing subset of the ACL2 language without guards. AIJ consists of (i) a Java representation of the ACL2 values, terms, and environment, (ii) a Java implementation of the ACL2 primitive

Shilpi Goel and Matt Kaufmann (Eds.):

ACL2 Theorem Prover and its Applications (ACL2-2018)

EPTCS 280, 2018, pp. 1-17 doi 10.4204/EPTCS.280.1
(C) Alessandro Coglio

This work is licensed under the Creative Commons Attribution License. 
functions, and (iii) an ACL2 evaluator written in Java. AIJ executes the deeply embedded Java representations of ACL2 functions generated by ATJ. AIJ is of independent interest and can be used without ATJ.

The ACL2 language subset supported by ATJ and AIJ includes all the values, all the primitive functions, and many functions with raw Lisp code-see Section 2 for details on these two kinds of functions.

The initial implementation of AIJ favored assurance over efficiency: it was quite simple, to reduce the chance of errors and facilitate its potential verification, but it was also quite slow. The careful introduction of a few optimizations, which do not significantly complicate the code but provide large speed-ups, makes the speed of the current implementation arguably adequate for some applications; see Section 5 . Furthermore, the code is amenable to additional planned optimizations; see Section 6 .

\section{Background: The Evaluation Semantics of ACL2}

ACL2 has a precisely defined logical semantics [11], expressed in terms of syntax, axioms, and inference rules, similarly to logic textbooks and other theorem provers. This logical semantics applies to logicmode functions, not program-mode functions. Guards are not part of the logic, but engender proof obligations in the logic when guard verification is attempted.

ACL2 also has a documented evaluation semantics [2, : doc evaluation], which could be formalized in terms of syntax, values, states, steps, errors, etc., as is customary for programming languages. This evaluation semantics applies to both logic-mode and program-mode functions. Guards affect the evaluation semantics, based on guard-checking settings. Even non-executable functions (e.g. introduced via defchoose or defun-nx) degenerately have an evaluation semantics, because they do yield error results when called; however, the following discussion focuses on executable functions.

Most logic-mode functions have definitions that specify both their logical and their evaluation semantics: for the former, the definitions are logically conservative axioms; for the latter, the definitions provide "instructions" for evaluating calls of the function. For a defined logic-mode function, the relationship between the two semantics is that, roughly speaking, evaluating a call of the function yields, in a finite number of steps, the unique result value that, with the argument values, satisfies the function's defining axiom - the actual relationship is slightly more complicated, as it may involve guard checking.

The primitive functions [2, : doc primitive] are in logic mode and have no definitions; they are all built-in. Examples are equal, if, cons, car, and binary-+. Their logical semantics is specified by axioms of the ACL2 logic. Their evaluation semantics is specified by raw Lisp code (under the hood). The relationship between the two semantics is as in the above paragraph, with the slight complication that pkg-witness and pkg-imports yield error results when called on unknown package names. The evaluation of calls of if is non-strict, as is customary.

Most program-mode functions have definitions that specify their evaluation semantics, similarly to the non-primitive logic-mode functions discussed above. Their definitions specify no logical semantics.

The logic-mode functions listed in the global variable logic-fns-with-raw-code have a logical semantics specified by their ACL2 definitions, but an evaluation semantics specified by raw Lisp code. (They are disjoint from the primitive functions, which have no definitions.) For some of these functions, e.g. len, the raw Lisp code just makes them run faster but is otherwise functionally equivalent to the ACL2 definitions. Others have side effects, carried out by their raw Lisp code but not reflected in their ACL2 definitions. For example, hard-error prints a message on the screen and immediately terminates execution, unwinding the call stack. As another example, fmt-to-comment-window prints a message on the screen, returning nil and continuing execution. But the ACL2 definitions of both of these example 
functions just return nil.

The program-mode functions listed in the global variable program-fns-with-raw-code have an evaluation semantics specified by raw Lisp code. Their ACL2 definitions appear to have no actual use.

Since stobjs [2, : doc stobj] are destructively updated, functions that manipulate stobjs may have side effects as well —namely, the destructive updates. Because of single-threadedness, these side effects are invisible in the end-to-end input/output evaluation of these functions; however, they may be visible in some formulations of the evaluation semantics, such as ones that comprehend interrupts, for which updating a record field in place involves different steps than constructing a new record value with a changed field. The built-in state stobj [2, : doc state $]$ is "linked" to external entities, e.g. the file system of the underlying machine. Thus, functions that manipulate state may have side effects on these external entities. For example, princ\$ (a member of logic-fns-with-raw-code) writes to the stream associated with the output channel argument, and affects the file system.

The fact that the side effects of the evaluation semantics are not reflected in the logical semantics is a design choice that makes the language more practical for programming while retaining the ability to prove theorems. But when generating Java or other code, these side effects should be taken into consideration: for instance, turning hard-error and fmt-to-comment-window into Java code that returns (a representation of) nil, would be incorrect or at least undesired. As an aside, a similar issue applies to the use of APT transformations [3]: for instance, using the simplify transformation [6] to turn calls of hard-error into nil, while logically correct and within simplify's stipulations, may be undesired or unexpected.

\section{AIJ: The Deep Embedding}

AIJ is a Java package whose public classes and methods provide an API to (i) build and unbuild representations of ACL2 values 11 (ii) build representations of ACL2 terms and of an ACL2 environment, and (iii) evaluate calls of ACL2 primitive and defined functions, without checking guards. By construction, the ACL2 code represented and evaluated by AIJ is executable, has no side effects, does not access stobjs, and has no guards.

AIJ consists of a few thousand lines of Java code (including blank and comment lines), thoroughly documented with Javadoc comments. The implementation takes advantage of object-oriented features like encapsulation, polymorphism, and dynamic dispatch.

The Java classes that form AIJ are shown in the simplified UML class diagram in Figure 1 and described in the following subsections. Each class is depicted as a box containing its name ${ }^{2}$ Abstract classes have italicized names. Public classes have names preceded by + , while package-private classes have names preceded by $\sim$. Inheritance ('is a') relationships are indicated by lines with hollow triangular tips. Composition ('part of') relationships are indicated by lines with solid rhomboidal tips, annotated with the names of the containing class instances' fields that store the contained class instances, and with the multiplicity of the contained instances for each containing instance ('0..' means 'zero or more').

\footnotetext{
${ }^{1}$ When talking about AIJ, this paper calls 'build' and 'unbuild' what is often called 'construct' and 'destruct' in functional programming, because in object-oriented programming the latter terms may imply object allocation and deallocation, which is not necessarily what the AIJ API does.

${ }^{2}$ In AIJ's actual code, each class name is prefixed with 'Acl2' (e.g. Acl2Value), so that external code can reference these classes unambiguously without AIJ's package name edu.kestrel.acl2.aij. This paper omits the prefix for brevity, and uses fully qualified names for the Java standard classes to avoid ambiguities, e.g. java.lang. String is the Java standard string class, as distinguished from String in Figure 1
} 


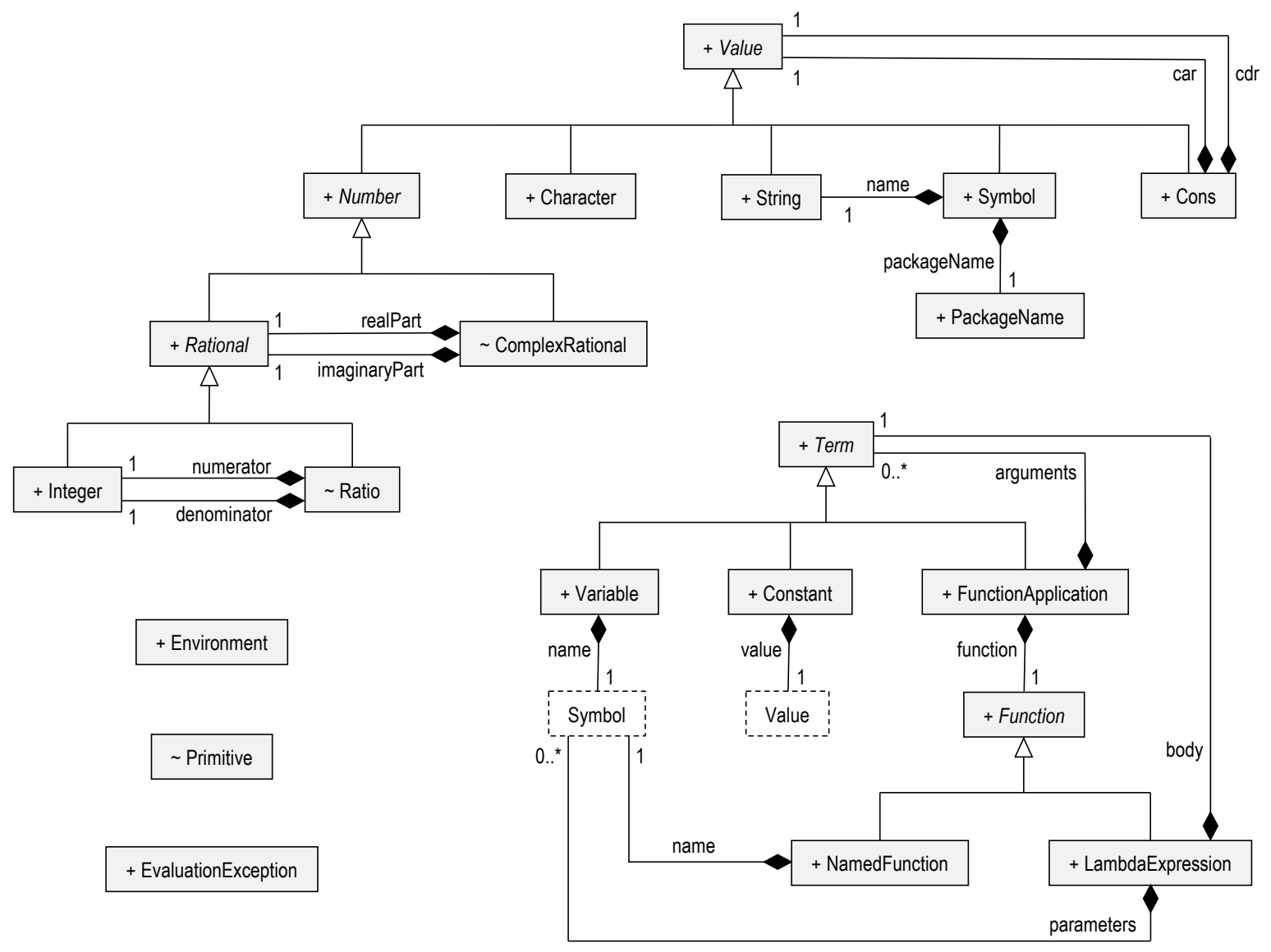

Figure 1: Simplified UML class diagram for AIJ.

The dashed boxes are just replicas to avoid clutter. This UML class diagram is simplified because the class boxes do not contain fields and methods.

\subsection{Values}

The set of values of the ACL2 evaluation semantics is the union of the sets depicted in Figure 2 (i) integers, recognized by integerp; (ii) ratios, i.e. rationals that are not integers, with no built-in recognizer $3^{3}$ (iii) complex rationals, recognized by complex-rationalp; (iv) characters, recognized by characterp; (v) strings, recognized by stringp; (vi) symbols, recognized by symbolp; and (vii) cons pairs, recognized by consp. Integers and ratios form the rationals, recognized by rationalp. Rationals and complex rationals form the Gaussian rationals, which are all the numbers in ACL2, recognized by ac12-numberp.4 The logical semantics of ACL2 allows additional values called 'bad atoms', and consequently cons pairs that may contain them directly or indirectly; however, such values cannot be constructed in evaluation.

AIJ represents ACL2 values as immutable objects of Value and its subclasses in Figure 1. Each

\footnotetext{
${ }^{3}$ The term 'ratio' is used in the Common Lisp specification [14 Section 2.1.2].

${ }^{4}$ This discussion does not apply to ACL2(r).
} 


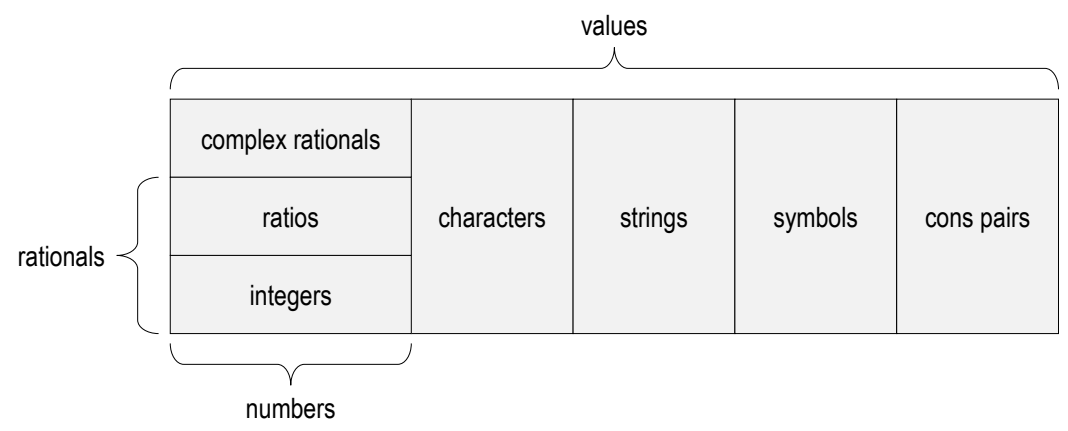

Figure 2: Values of the ACL2 evaluation semantics.

such class corresponds to a set in Figure 2. The subset relationships in Figure 2 match the inheritance relationships in Figure 1. The sets of values that are unions of other sets of values correspond to abstract classes; the other sets correspond to concrete classes. All these classes are public, except for the packageprivate ones for ratios and complex rationals: ratios and complex rationals are built indirectly via AIJ's API, by building rationals that are not integers and numbers that are not rationals.

The information about the represented ACL2 values is stored in fields of the non-abstract classes. Integer stores the numeric value as a java.math.BigInteger. Ratio stores the numerator and denominator as Integers, in reduced form (i.e. their greatest common divisor is 1 and the denominator is greater than 1). ComplexRational stores the real and imaginary parts as Rationals. Character stores the 8-bit code of the character as a char below 256. String stores the codes and order of the characters as a java.lang. String whose chars are all below 256. Symbol stores the symbol's package name as a PackageName (a wrapper of java.lang. String that enforces the ACL2 constraints on package names) and the symbol's name as a String. Cons stores the component Values. All these fields are private, thus encapsulating the internal representation choices and enabling their localized modification. ACL2 numbers, strings, and symbols have no preset limits, but the underlying Lisp runtime may run out of memory. Their Java representations (e.g. java .math. BigInteger) have very large limits, whose exceedance could be regarded as running out of memory. If needed, the Java representations could be changed to overcome the current limits (e.g. by using lists of java.math.BigIntegers).

The public classes for ACL2 values and package names provide public static factory methods to build objects of these classes. For example, Character.make(char) returns a Character with the supplied argument as code, throwing an exception if the argument is above 255. As another example, Cons.make (Value, Value) returns a Cons with the supplied arguments as components. Some classes provide overloaded variants, e.g. Integer make (int) and Integer make (java.math. BigInteger). All these classes provide no public Java constructors, thus encapsulating the details of object creation and re-use, which is essentially transparent to external code because these objects are immutable.

The public classes for ACL2 values provide public instance getter methods to unbuild (i.e. extract information from) objects of these classes. For example, Character.getJavaChar () returns the code of the character as a char that is always below 256. As another example, Cons.getCar() and Cons.getCdr () return the component Values of the cons pair. Some classes provide variants, e.g. Integer.getJavaInt() (which throws an exception if the integer does not fit in an int) and Integer get JavaBigInteger(). 


\subsection{Terms}

ACL2 translates the terms supplied by the user, which may include macros and named constants, into a restricted internal form, in which macros and named constants are expanded [2, :doc term]. In the rest of this paper, 'term' means 'translated term', i.e. a term in the restricted internal form.

The set of ACL2 terms consists of (i) variables, which are symbols, (ii) quoted constants, which are lists (quote value) where value is a value, and (iii) function applications, which are lists ( $f n \arg 1$ $\ldots \quad a r g_{n}$ ) where $f n$ is a function and $a r g_{1}, \ldots, \arg _{n}$ are zero or more terms. A function $f n$ used in a term is (i) a named function, which is a symbol, or (ii) a lambda expression, which is a list (lambda $\left(v_{v a} \ldots v_{1} \ldots v r_{m}\right)$ body) where $v a r_{1}, \ldots, v a r_{m}$ are zero or more symbols and body is a term, whose free variables are all among $v a r_{1}, \ldots, v a r_{m}$ (i.e. lambda expressions are always closed).

AIJ represents ACL2 terms in a manner similar to ACL2 values, as immutable objects of Term and its subclasses in Figure 1; functions are represented as immutable objects of Function and its subclasses in Figure 1. The superclasses are abstract, while the subclasses are concrete. All these classes are public.

The information about the represented ACL2 terms is stored in private fields of the non-abstract classes. Variable and NamedFunction are wrappers of Symbol. Constant is a wrapper of Value 5 FunctionApplication stores a Function and an array of zero or more Terms. LambdaExpression stores an array of zero or more Variables and a Term.

The non-abstract classes for ACL2 terms (and functions) provide public static factory methods to build objects of these classes, but no public Java constructors, similarly to the classes for ACL2 values.

\subsection{Environment}

ACL2 terms are evaluated in an environment that includes function definitions, package definitions, etc. AIJ stores information about part of this environment in Environment in Figure 1 . Since there is just one environment at a time in ACL2, this class has no instances and only static fields and methods.

An ACL2 function definition consists of several pieces of information, of which AIJ only stores (i) the name, which is a symbol, (ii) the parameters, which are zero or more symbols, and (iii) the body, which is a term. Environment stores function definitions in a private static field, as a java.util. Map from Symbols for the functions' names to LambdaExpressions for the functions' parameters and bodies. The public static method Environment.addFunctionDef (Symbol, Symbol [] , Term) adds a function definition to the map.

An ACL2 package definition associates a list of imported symbols to a package name. Environment stores package definitions in a private static field, as a java.util. Map from PackageNames for the packages' names to java.util.Lists of Symbols for the packages' import lists. The public static method Environment.addPackageDef (PackageName, List<Symbol>) adds a package definition to the map. AIJ uses this field to implement the primitive function pkg-imports. AIJ also uses information derived from this field to implement the overloaded factory methods Symbol .make that build symbols: for instance, Symbol .make ("ACL2", "CONS") returns a Symbol with name "CONS" and package name "COMMON-LISP", not package name "ACL2", because "ACL2" imports cons from "COMMON-LISP"; the call Symbol.make ("ACL2", "CONS") is the Java equivalent of the ACL2 symbol notation ac12: : cons.

Environment also stores the value of the ACL2 constant *pkg-witness-name* in a private static field, as a java.lang. String. This field may be set, at most once (otherwise an exception is thrown),

\footnotetext{
${ }^{5}$ These wrappers place Symbols and Values into the class hierarchy of Term and Function, given that Java does not support multiple class inheritance. For instance, Symbol could not be both a subclass of Value and a subclass of Term.
} 
via the public static method Environment.setPackageWitnessName (java.lang. String). AIJ uses this field to implement the primitive function pkg-witness.

\subsection{Primitive Functions}

Since the ACL2 primitive functions have no definitions, AIJ cannot evaluate their calls via their bodies as described in Section 3.5. AIJ implements these functions "natively" in Java, in the package-private class Primitive in Figure 11. Each primitive function except if, whose calls are evaluated non-strictly as described in Section 3.5, is implemented by a private static method Primitive.execPrim (Value) or Primitive.execPrim (Value, Value) (based on the function's arity), where Prim is a Java "version" of the function's name; the method returns a Value. For instance, Primitive. execCharCode (Value) implements char-code. The package-private static method Primitive.call (Symbol, Value []) evaluates a call of the primitive function named by the Symbol argument on the values in the Value [] argument, by calling the appropriate Primitive.execPrim method and returning the result Value. Primitive has no fields and only the above static methods; no instances of this class are created.

The recognizers integerp, consp, etc. are implemented to return a Symbol for $t$ or nil, based on whether the argument Value is an instance of Integer, Cons, etc.

The destructors car, numerator, etc. are implemented to return information from the private fields.

The constructors complex and cons are implemented via the factory methods Number.make and Cons.make. The constructor intern-in-package-of-symbol is implemented via the factory method Symbol .make, and it also calls the getter method Symbol. getPackageName on the second argument.

The conversions char-code and code-char are implemented by passing information from the private field of one class to the factory method of the other class. The conversion coerce has a slightly more laborious implementation, which scans or builds a Java representation of an ACL2 list.

The arithmetic operation unary-- is implemented via package-private instance methods negate() in the numeric classes. Primitive. execUnaryMinus, when given $x$ as argument, calls $x$.negate(). Dynamic dispatch selects a negate method based on the runtime class of $x$. Integer negate calls java.math.BigInteger.negate on its private field and uses the result to return a negated Integer. Since $-(a / b)=(-a) / b$, Ratio.negate calls Integer.negate on the numerator, and uses the result to return a negated Ratio. Since $-(a+b i)=(-a)+(-b) i$, ComplexRational. negate calls Rational .negate on the real and imaginary parts, and uses the returned Rationals to return a negated ComplexRational; each of these two calls to Rational .negate is, in turn, dynamically dispatched to Integer.negate or Ratio. negate, based on the runtime classes of the real and imaginary parts.

The arithmetic operation binary-+ is similarly implemented via package-private instance methods add (Value) in the numeric classes. But the presence of the second argument leads to a slightly more complicated interplay among the methods. Primitive. execBinaryPlus, when given $x$ and $y$ as arguments, calls $x$. add $(y)$, dynamically dispatching based on the runtime class of $x$. Integer add splits into two cases: if $y$ is an Integer, an Integer sum is returned using java.math.BigInteger.add; otherwise, the roles of $x$ and $y$ are swapped, exploiting the commutativity of addition, by calling $y$. add $(x)$, which dynamically dispatches to a different add method. Ratio.add performs an analogous split; since $a / b+c / d=(a d+c b) / b d$, this method calls the multiply methods, further complicating the method interplay. Since $(a+b i)+(c+d i)=(a+c)+(b+d) i$, ComplexRational.add calls Rational.add on the real and imaginary parts, which are further dynamically dispatched to Integer . add or Ratio. add.

The arithmetic operations unary-/ and binary-* are implemented analogously to unary-- and binary-+, via reciprocate() and times(Value) methods in the numeric classes. There is some 
additional interplay among methods: for instance, since $1 /(a+b i)=\left(a /\left(a^{2}+b^{2}\right)\right)-\left(b /\left(a^{2}+b^{2}\right)\right) i$, ComplexRational . reciprocate calls all the arithmetic methods of Rational.

The arithmetic comparison < is implemented analogously to binary-+ and binary-*, as part of an implementation of ACL2's total order [2, : doc lexorder] via compareTo(Value) methods in Value and its subclasses, which all implement the java.lang. Comparable<Value $>$ interface. In the compareTo methods in the numeric classes, when the roles of $x$ and $y$ are swapped in the same way as in the add methods described above, the result is negated before being returned, because comparison, unlike addition and multiplication, is not commutative.

The equality equal is implemented via methods equals (java.lang.Object) in Value and its subclasses, which override java.lang.Object.equals. These equality methods are implemented in the obvious way.

The implementation of bad-atom<= returns a Symbol for nil, consistently with the raw Lisp code.

The functions pkg-imports and pkg-witness are implemented as discussed in Section 3.3. They throw an exception if the argument does not name a defined package, matching ACL2's behavior.

All of these implementations do not check guards. They handle Values outside the guards according to the applicable ACL2 completion axioms.

\subsection{Evaluation}

AIJ evaluates ACL2 terms via (i) eval (java.util. Map<Symbol, Value $>$ ) methods in Term and its subclasses, and (ii) apply (Value [] ) methods in Function and its subclasses. This evaluation approach is well known [12].

The eval methods take maps as arguments that bind values to variable symbols, and return Value results. Constant. eval returns the constant's value, ignoring the map. Variable. eval returns the value bound to the variable, throwing an exception if the variable is unbound. Application.eval recursively evaluates the argument terms and then calls Function.apply on the function and resulting values. However, if the function represents if, Application. eval first evaluates just the first argument, and then, based on the result, either the second or third argument, consistently with the non-strictness of if.

The apply methods take arrays of zero or more Values as arguments, and return Value results. LambdaExpression. apply evaluates the lambda expression's body with a freshly created map that binds the values to the parameters - no old bindings are needed, because lambda expressions are closed. NamedFunction. apply calls a public method Environment.call (Symbol, Value []) with the name of the function and the argument values. Environment. call operates as follows: if the symbol names a primitive function, it is forwarded, with the values, to Primitive.call; if the symbol names a function defined in the environment, the lambda expression that defines the function is applied to the values; if the symbol does not name a primitive or defined function, an exception is thrown.

AIJ evaluates ACL2 terms in a purely functional way, without side effects ${ }^{6}$ AIJ does not check the guards of primitive or defined functions. The aforementioned method Environment. call calls ACL2 functions on values only, not on (names of) stobjs.

\subsection{Usage}

AIJ is designed to be used as follows by Java code outside AIJ's package:

\footnotetext{
${ }^{6}$ Aside from exhausting the available memory, which is, unavoidably, always a possibility.
} 
1. Define all the ACL2 packages of interest by repeatedly calling Environment.addPackageDef. For each package, use the factory methods of PackageName and Symbol to build the name and the imported symbols. Define both the built-in and user-defined packages, in the order in which they appear in the ACL2 history. This order ensures that Symbol.make does not throw an exception due to an unknown package.

2. Define all the ACL2 functions of interest by repeatedly calling Environment.addFunctionDef. For each function, use the factory methods of the value and term classes to build the name, the parameters, and the body. The functions can be defined in any order, so long as all the packages are defined before the functions (see step above).

3. Call Environment. setPackageWitnessName with the appropriate value from the ACL2 constant $*$ pkg-witness-name*.

4. Call an ACL2 primitive or defined function as follows:

(a) Build the name of the ACL2 function to call, as well as zero or more ACL2 values to pass as arguments, via the factory methods of the value classes.

(b) Call Environment.call with the Symbol that names the function and the Value array of arguments.

(c) Unbuild the returned Value as needed to inspect and use it, using the getter methods of the value classes.

5. Go back to step 4 as many times as needed.

The above protocol explains why AIJ provides a public API for unbuilding ACL2 values but no public API to unbuild the other ACL2 entities (terms etc.). The latter are built entirely by Java code outside AIJ's package, which therefore has no need to unbuild the entities that it builds. Values, instead, may be built by executing ACL2 code that returns them as results: Java code outside AIJ's package may need to unbuild the returned values to inspect and use them.

Besides the structural constraints implicit in the Java classes, and the existence of the referenced packages when building symbols (necessary to resolve imported symbols), AIJ does not enforce any well-formedness constraints when building terms and other entities, e.g. the constraint that the number of arguments in a function call matches the function's arity. However, during evaluation, AIJ makes no well-formedness assumptions and performs the necessary checks, throwing informative exceptions if these checks fail.

\section{ATJ: The Code Generator}

ATJ is an ACL2 tool that provides an event macro to generate Java code from specified ACL2 functions. The generated Java code provides a public API to (i) build an AIJ representation of the ACL2 functions and other parts of the ACL2 environment and (ii) evaluate calls of the functions on ACL2 values via AIJ. The Java code generated by ATJ automates steps 1, 2, and 3 in Section 3.6 and provides a light wrapper for step $4 \mathrm{~b}$, while steps $4 \mathrm{a}$ and $4 \mathrm{c}$ must be still performed directly via AIJ's API.

ATJ consists of a few thousand lines of ACL2 code (including blank lines, implementation-level documentation, and comments), accompanied by a few hundred lines of user-level documentation in XDOC. The implementation is thoroughly documented in XDOC as well.

\subsection{Overview}

ATJ generates a single Java file containing a single class, with the following structure: 


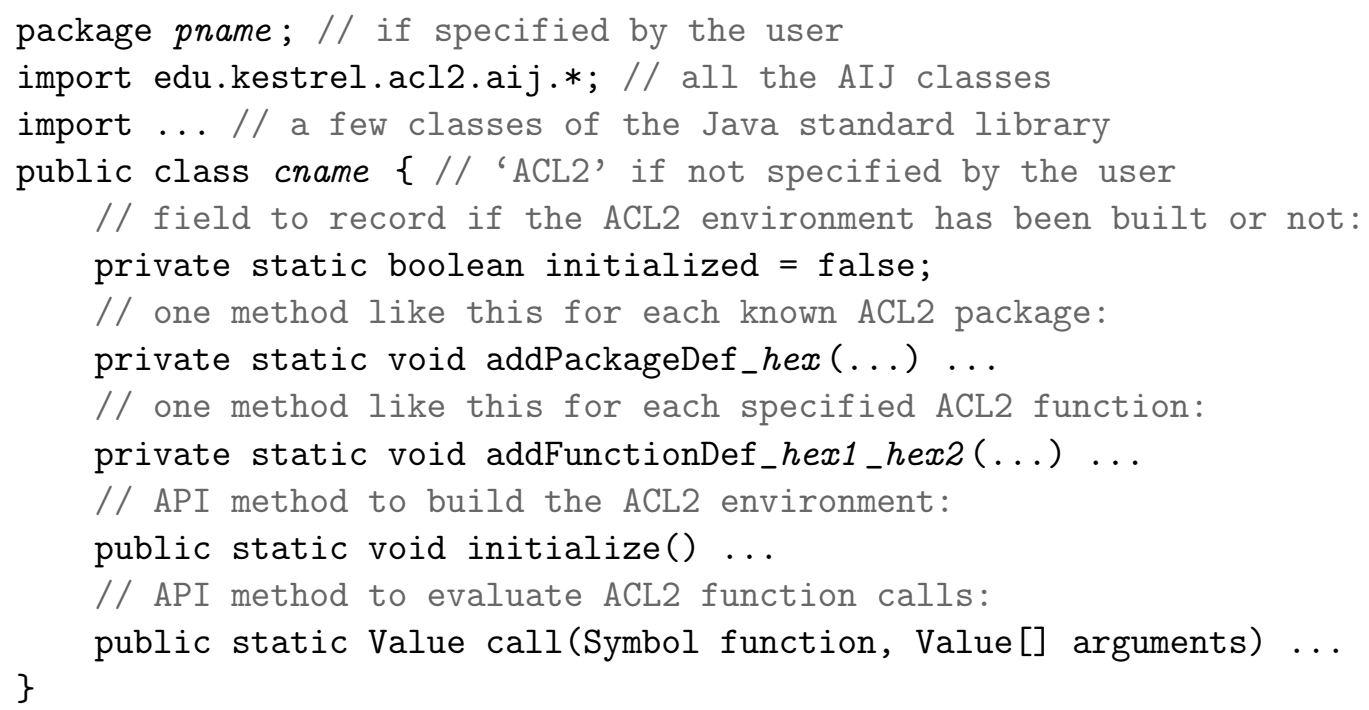

The file has the same name as the class; it is (over)written in the current working directory, unless the user specifies a directory. ATJ directly generates Java concrete syntax, via formatted printing to the ACL2 output channel associated to the file, without going through a Java abstract syntax and pretty printer.

\subsection{Value and Term Building}

As part of building an AIJ representation of the ACL2 environment, the Java code generated by ATJ builds AIJ representations of ACL2 values and terms: function definitions include terms as bodies, and constant terms include values. It does so via the factory methods discussed in Sections 3.1 and 3.2.

In principle, ATJ could turn each ACL2 value or term into a single Java expression with an "isomorphic" structure. For example, the ACL2 value ((10 . \#\A) . "x") could be built as follows:

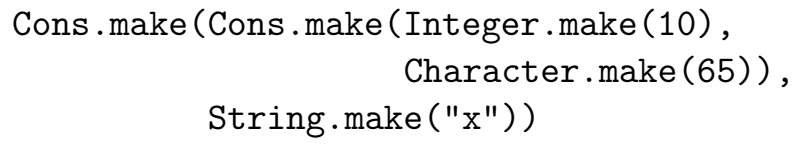

However, values and terms of even modest size (e.g. function bodies) would lead to large expressions, which are not common in Java. Thus, ATJ breaks them down into sub-expressions assigned to local variables. For instance, the example value above is built as follows:

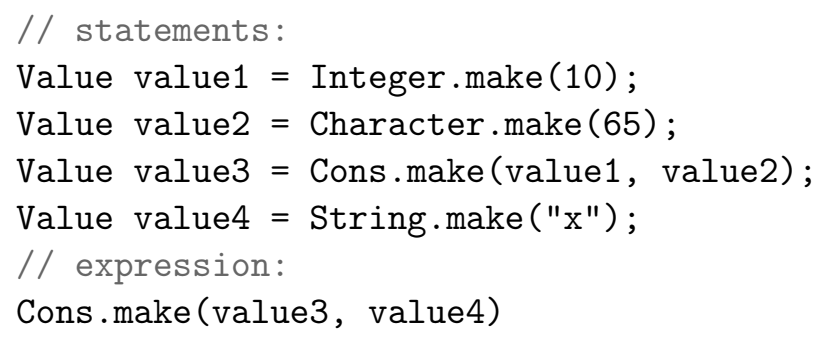

In general, ATJ turns each ACL2 value or term into (i) zero or more Java statements that incrementally build parts of it and (ii) one Java expression that builds the whole of it from the parts. ATJ does so recursively: the expression for a sub-value or sub-term is assigned to a new local variable that is used in the expression for the containing super-value or super-term. The top-level expressions are used as explained in Sections 4.3 and 4.4 . 
To generate new local variable names, ATJ keeps track of three numeric indices (for values, terms, and lambda expressions - recall that the latter are mutually recursive with terms) as it recursively traverses values and terms. The appropriate index is appended to 'value', 'term', or 'lambda' and then incremented.

\subsection{Package Definition Building}

The Java code generated by ATJ builds an AIJ definition of each ACL2 package known when ATJ is invoked. The names of the known packages are the keys of the alist returned by the built-in function known-package-alist, in reverse chronological order.

The AIJ definition of each of these packages is built by a method addPackageDef_hex (see Section 4.1), where hex is an even-length sequence of hexadecimal digits for the ASCII codes of the characters that form the package name. For instance, the definition of the "ACL2" package is built by addPackageDef_41434C32. This simple naming scheme ensures that the generated method names are distinct and valid, since ACL2 package names allow characters disallowed by Java method names.

Each addPackageDef_hex method builds a Java list of all the symbols imported by the package, which ATJ obtains via pkg-imports. Then the method calls Environment.addPackageDef with the PackageName and the list of Symbols.

\subsection{Function Definition Building}

The Java code generated by ATJ builds an AIJ definition of each (non-primitive) ACL2 function specified via one or more function symbols $f n_{1}, \ldots, f n_{p}$ supplied to ATJ. Each $f n_{i}$ implicitly specifies not only $f n_{i}$ itself, but also all the functions called directly or indirectly by $f n_{i}$, ensuring the "closure" of the generated Java code under ACL2 calls.

ATJ uses a worklist algorithm, initialized with $\left(f n_{1} \ldots f n_{p}\right)$, to calculate a list of their closure under calls. Each iteration removes the first function $f n$ from the worklist, adds it to the result list, and extends the worklist with all the functions directly called by $f n$ that are not already in the result list. Here 'directly called by' means 'occurring in the unnormalized-body property of'; occurrences in the guard of $f n$ do not count, because ATJ, like AIJ, ignores guards. If $f n$ has no unnormalized-body property, it must be primitive, otherwise ATJ stops with an error-this happens if $f n$ is a constrained, not defined, function. If $f n$ is in logic-fns-with-raw-code or program-fns-with-raw-code (see Section 22, it must be in a whitelist of functions that are known to have no side effects.7 If $f n$ has input or output stobjs, ATJ stops with an error-this may only happen if $f n$ is not primitive.

The AIJ definition of each of these functions is built by a method addFunctionDef_hex1_hex2 (see Section 4.1), where hex1 and hex2 are even-length sequences of hexadecimal digits for the ASCII codes of the package and symbol names of the function symbol. For instance, the definition of the len function is built by addFunctionDef_41434C32_4C454E. This simple naming scheme ensures that the generated method names are distinct and valid, since ACL2 package and symbol names allow characters disallowed by Java method names.

Each addFunctionDef_hex1_hex2 method first builds a Term from the unnormalized-body property of the function, as explained in Section 4.2. Then the top-level Java expression, along with a Symbol for the function name and with a Variable array for the function parameters, is passed to Environment.addFunctionDef.

\footnotetext{
${ }^{7}$ This whitelist is currently a subset of logic-fns-with-raw-code. It consists of functions whose raw Lisp code makes them run faster but is otherwise functionally equivalent to the ACL2 definitions.
} 


\subsection{Environment Building}

The initialize method generated by ATJ (see Section 4.1) calls all the addPackageDef_hex and addFunctionDef_hex1_hex2 methods described in Sections 4.3 and 4.4. The method also calls Environment. setPackageWitnessName with an argument derived from *pkg-witness-name*.

The package definition methods are called in the same order in which the corresponding packages are defined, which is the reverse order of the alist returned by known-package-alist.

This ensures the success of the calls of Symbol.make that build the elements of a package's import list. For instance, if "P" imports $q:$ : sym, then "Q" must be already defined when "P" is being defined. That is, addPackageDef_51 must have already been called when addPackageDef_50 calls Symbol .make ("Q", "SYM") as part of building "P"'s import list, which is needed to define "P"; otherwise, Symbol .make ("Q", "SYM") would throw an exception due to "Q" being still undefined.

The function definition methods are called after the package definition methods, again to ensure the success of the Symbol . make calls. The relative order of the function definitions is unimportant; the result list returned by ATJ's worklist algorithm (see Section 4.4) is in no particular order.

The initialize method may be called once by external code: the method throws an exception unless the initialized field (see Section 4.1) is false, and sets the field to true just before returning.

\subsection{Call Forwarding}

The cname . call method generated by ATJ (see Section 4.1) forwards the function name and the argument values to Environment.call, after ensuring that the initialized field is true, i.e. that the ACL2 environment has been built. It throws an exception if initialized is still false.

\section{Preliminary Tests and Optimizations}

The initial version of AIJ was deliberately written in a very simple way, without regard to performance, as a sort of "executable specification" in Java. The reasons were to increase assurance by reducing the chance of errors, facilitate the potential verification of the code, avoid premature optimizations, and observe the impact of gradually introduced optimizations.

Performance has been tested mainly on three example programs. The first is an ACL2 function that computes factorial non-tail-recursively. The second is an ACL2 function that computes Fibonacci non-tail-recursively. The third is a slightly modified version of the verified ABNF grammar parser [5] from the ACL2 Community Books [2, :doc abnf::grammar-parser]: the parser, in the :logic part of mbe, calls nat-list-fix on its input list of natural numbers just before reading each natural number, which makes execution "in the logic" (which is how AIJ executes) unduly slow; for testing AIJ more realistically, the parser was tweaked to avoid these calls of nat-list-fix. The tweaked parser is about 2,000 lines (including blank lines), including theorems to prove its termination so that it is in logic mode, and including return type theorems to prove its guards. The parser not only recognizes ABNF grammars, but also returns parse trees ${ }^{8}$

Unsurprisingly, the initial version of AIJ was quite slow. A re-examination of the code from a performance perspective readily revealed several easy optimization opportunities, which were carried out and are part of the current version of AIJ. These are the main ones, in order:

\footnotetext{
${ }^{8}$ Initially, tests were conducted on a simplified version of the parser that only recognized ABNF grammars, because ATJ did not support mbe, which is used in the construction of parse trees (defined via fixtypes [2, doc fty]). After extending ATJ to support mbe, testing was switched to the more realistic version of the parser that also returns parse trees.
} 
1. The Character array representation of Strings was replaced with java. lang. String.

2. Symbols frequently used during evaluation, such as the ones for $t$, nil, and the names of the primitive functions, were cached as constants instead of being built repeatedly.

3. Characters were interned, as follows. Character objects for all the 256 codes were pre-created and stored into an array, in the order of their codes. The factory method Character.make (char) indexes the array with the input code and returns the corresponding object. Since this ensures that there is just one object for each character code, Character . equals uses pointer equality (i.e. ==) and the default fast java.lang. Object.hashCode is inherited.

4. PackageNames, Strings, and Symbols were interned, similarly to Characters, as follows. Since there is a potentially infinite number of them, they are are created on demand. For each of these three classes, all the objects created thus far are stored as values of a java.util.Map, whose keys are java.lang. Strings for PackageName and String, and PackageNames paired with Strings for Symbol-the pairing is realized via nested maps. Each factory method first consults the appropriate map, either returning the existing object, or creating a new one that is added to the map. Similarly to Character's interning, the interning of these classes enables the use of pointer equality in the equality methods and the inheritance of the default fast hash code method.

Thanks to AIJ's object-oriented encapsulation, all these optimizations were easy and localized. These optimizations did not involve ATJ, because the code generated by ATJ is essentially used just to initialize the ACL2 environment (see Section 4.5), which happens quickly for the factorial and Fibonacci functions and for the ABNF parser.

Based on a few time measurements on the ABNF parser and a few other artificial programs, the above optimizations reduced execution time, very roughly, by the following factors, one after the other: 2 for optimization \#1, 5 for optimization \#2, and 2 for optimizations \#3 and \#4-all combined, 20.

Tables 1, 2, and 3 report more systematic time measurements for the factorial function, Fibonacci function, and ABNF parser. Each row corresponds to an input of the program: natural numbers for the factorial and Fibonacci functions; ABNF grammars (all from Internet standards, including ABNF itself) for the ABNF parser. The 'ACL2' columns are for execution in ACL2, with guard checking ('g.c.') set to t, i.e. typical execution, and :none, i.e. execution "in the logic"; the latter matches AIJ's execution. The "AIJ' column is for execution with AIJ's current version. Each cell contains minimum, average, and maximum real times from 10 runs, in seconds rounded to the millisecond. The ACL2 times were measured as the difference between the results of read-run-time just before and just after the call of the factorial function, Fibonacci function, or top-level ABNF parsing function. The Java times were measured as the difference between the results of java.lang. System.currentTimeMillis() just before and just after the call of cname . call on the corresponding ACL2 function. Given the AIJ evaluator's recursive implementation, a larger stack size than the default must be passed to the JVM (1 GB for these time measurements) to avoid a stack overflow.

The times in Table 1 are all roughly comparable for each input, with ACL2 faster on smaller inputs and AIJ faster on larger inputs: presumably, most of the time is spent multiplying large numbers, which all happens in java.math. BigInteger in the Java code and in Lisp's bignum implementation in the ACL2 code, dwarfing the contributions of ACL2 and AIJ proper, especially for the larger inputs ${ }^{9}$ The times in Table 2 differ: looking at the averages, AIJ is about 17-30 times slower than ACL2 with guard checking : none, which is about 8-10 times slower than ACL2 with guard checking t. The times in Table 3 differ as well: looking at the averages, AIJ is about 19-22 times slower than ACL2 with guard checking : none, which is about 16-87 times slower than ACL2 with guard checking t; nonetheless,

\footnotetext{
${ }^{9}$ Even the initial, unoptimized version of AIJ took comparable times.
} 
the absolute times suggest that the Java code of the parser is usable ${ }^{10}$ Performance needs vary: AIJ's current speed may be adequate to some applications, such as security-critical interactive applications like cryptocurrency wallets. Furthermore, as discussed in Section 6, there are more opportunities to optimize AIJ.

\begin{tabular}{|r||ccc|ccc|ccc|}
\hline \multicolumn{1}{|c||}{ Input } & \multicolumn{2}{|c|}{ ACL2 [g.c. t] } & \multicolumn{2}{c|}{ ACL2 [g.c. : none] } & \multicolumn{3}{c|}{ AIJ } \\
& min & avg & max & min & avg & max & min & avg & max \\
\hline \hline 1,000 & 0.000 & 0.000 & 0.001 & 0.000 & 0.000 & 0.001 & 0.003 & 0.005 & 0.012 \\
\hline 5,000 & 0.007 & 0.011 & 0.022 & 0.007 & 0.009 & 0.011 & 0.009 & 0.029 & 0.059 \\
\hline 10,000 & 0.031 & 0.035 & 0.038 & 0.032 & 0.034 & 0.040 & 0.026 & 0.035 & 0.068 \\
\hline 50,000 & 1.324 & 1.355 & 1.432 & 1.319 & 1.328 & 1.337 & 0.589 & 0.687 & 1.044 \\
\hline 100,000 & 6.280 & 6.385 & 6.604 & 6.279 & 6.291 & 6.307 & 2.340 & 2.547 & 2.705 \\
\hline
\end{tabular}

Table 1: Time measurements for the factorial function.

\begin{tabular}{|r||ccc|ccc|ccr|}
\hline \multirow{2}{*}{ Input } & \multicolumn{2}{|c|}{ ACL2 [g.c. t] } & \multicolumn{2}{c|}{ ACL2 } & [g.c. : none] & \multicolumn{3}{c|}{ AIJ } \\
& min & avg & max & min & avg & max & min & avg & \multicolumn{1}{c|}{ max } \\
\hline \hline 10 & 0.000 & 0.000 & 0.000 & 0.000 & 0.000 & 0.000 & 0.000 & 0.001 & 0.004 \\
\hline 20 & 0.000 & 0.000 & 0.000 & 0.001 & 0.001 & 0.001 & 0.019 & 0.030 & 0.053 \\
\hline 30 & 0.007 & 0.008 & 0.009 & 0.061 & 0.063 & 0.079 & 1.043 & 1.094 & 1.210 \\
\hline 40 & 0.727 & 0.734 & 0.750 & 7.144 & 7.205 & 7.355 & 126.167 & 127.149 & 129.959 \\
\hline
\end{tabular}

Table 2: Time measurements for the Fibonacci function.

\begin{tabular}{|c|c|c|c|c|c|c|c|c|c|}
\hline \multirow{2}{*}{ Input } & \multicolumn{3}{|c|}{ ACL2 [g.c. t] } & \multicolumn{3}{|c|}{ ACL2 [g.c. : none] } & \multicolumn{3}{|c|}{ AIJ } \\
\hline & $\min$ & avg & $\max$ & $\min$ & avg & $\max$ & $\min$ & avg & $\max$ \\
\hline ABNF grammar & 0.004 & 0.007 & 0.014 & 0.109 & 0.113 & 0.117 & 2.391 & 2.478 & 3.076 \\
\hline JSON grammar & 0.001 & 0.002 & 0.006 & 0.044 & 0.049 & 0.053 & 1.011 & 1.023 & 1.031 \\
\hline URI grammar & 0.002 & 0.003 & 0.006 & 0.100 & 0.105 & 0.112 & 2.218 & 2.233 & 2.251 \\
\hline HTTP grammar & 0.002 & 0.004 & 0.010 & 0.167 & 0.175 & 0.189 & 3.577 & 3.597 & 3.626 \\
\hline IMF grammar & 0.009 & 0.014 & 0.021 & 1.028 & 1.079 & 1.414 & 21.173 & 21.522 & 21.741 \\
\hline SMTP grammar & 0.007 & 0.010 & 0.019 & 0.398 & 0.404 & 0.411 & 8.648 & 8.733 & 8.896 \\
\hline IMAP grammar & 0.020 & 0.026 & 0.030 & 2.198 & 2.267 & 2.481 & 43.083 & 43.490 & 43.805 \\
\hline
\end{tabular}

Table 3: Time measurements for the ABNF parser.

All the time measurements were taken on a MacBook Pro (15-inch, 2017) with $3.1 \mathrm{GHz}$ Intel Core i7 and 16 GB $2133 \mathrm{MHz}$ LPDDR3, running macOS High Sierra Version 10.13.6. The ACL2 times were measured with commit 852ee0aca96deac2b3c062ee03f458acca668f6e from GitHub running on 64-bit Clozure Common Lisp Version 1.11.5. The Java times were measured with the version of AIJ in the same commit from GitHub as above, running on Oracle's 64-bit Java 10 2018-03-20, Java SE Runtime Environment 18.3 (build 10+46). Just before taking the measurements, the machine was rebooted and only the necessary applications were started.

The performance of ATJ does not affect the performance of the Java code. ATJ runs in 1-2 seconds on each of the factorial function, Fibonacci function, and ABNF parser, including the time to write the Java files; this was measured by wrapping the calls of ATJ with time $\$$.

\footnotetext{
${ }^{10}$ As another data point, the simplified parser mentioned in Footnote 8 was about 4-7 times faster than the current parser.
} 


\section{Future Work}

Evaluating non-executable functions (i.e. non-primitive and without an unnormalized-body property), by throwing an exception that mirrors the error that ACL2 yields, is easy but not necessarily useful [11 A planned extension is to support guards and evaluation with different guard-checking settings, in the same way as ACL2. Support for functions with side effects will be added one at a time, by writing native Java implementations (as done for the primitive functions) that suitably mirror the ACL2 side effects in Java; for instance, hard errors could be implemented as exceptions. User-defined stobjs could be supported by storing their contents in Java fields that are destructively updated; since state is "linked" to external entities (e.g. the file system), support for this built-in stobj will involve the use of the Java API of those entities. Supporting stobjs also involves extending AIJ's public API to call ACL2 functions on stobj names, besides values. Direct support for calling macros directly, and for supplying named constants to function calls, are also candidate extensions.

The generated method cname . call described in Section 4.6 does not provide much beyond calling Environment. call directly, but is suggestive of additional functionality. For example, future versions of cname could provide a public method for each top-level target function $f n_{i}$ supplied to ATJ, with no parameter for the function name, and with as many Value parameters as the function's arity instead of a single Value array parameter. As another example, cname could provide additional public methods to call each $f n_{i}$ on objects of more specific types (e.g. Integer instead of Value), based on the guards. The names of these methods should be derived from the names of the corresponding functions, according to safe but more readable schemes than the one described in Section 4.4 - in fact, a more readable scheme should be used for the methods described in Section 4.3 and Section 4.4 as well.

A reviewer suggested to make the fields and methods of Environment non-static and have multiple instances of this class at once. This is worth exploring.

There are more optimization opportunities beyond the ones already carried out and described in Section 5. For example, now each variable evaluation looks up the variable symbol in the hash map that stores the current binding of values to variables. As another example, now each function call first looks up the function symbol in the hash map that stores the function definitions in the environment, and then, if no definition is found, it compares the function symbol with all the primitive function symbols until a match is found. Replacing or enhancing AIJ's representation of variable and function symbols with numeric indices should make all these accesses much faster ${ }^{12}$ As a third example, AIJ's evaluator could be re-implemented as a loop with an explicit stack, instead of a recursion. As a fourth example, many built-in ACL2 functions could be implemented natively in Java (as done for the primitive functions), instead of being interpreted.

A reviewer suggested to implement hons [2, :doc hons-and-memoization] in AIJ, and use it instead of cons. This amounts to interning all the Java objects that represent ACL2 values (not just Characters, Strings, and Symbols - see Section 5p, enabling fast equality tests and hash code computations, which could increase performance in some applications. Perhaps future versions of AIJ and ATJ could provide options to use hons vs. cons.

The eventual path to fast execution is to avoid the interpretation overhead, by having ATJ turn ACL2 functions into shallowly embedded Java representations, as is customary in code generators ${ }^{13}$ The shal-

\footnotetext{
${ }^{11}$ If support for evaluating non-executable function is added, ATJ should still include an option to signal an error when the worklist algorithm reaches a non-executable function.

${ }^{12}$ A preliminary experiment with just variable indices seems to reduce execution times roughly by 2 .

${ }^{13}$ Besides more conventional translation approaches, a more speculative idea is to generate the shallowly embedded representations by partially evaluating [10] the AIJ interpreter on the deeply embedded representations generated by ATJ.
} 
low embedding will consist of Java methods that implement the ACL2 functions, with suitably matching signatures. AIJ's representation of and operations on ACL2 values will still be used, but AIJ's representation and evaluator of ACL2 terms will not. Under certain conditions, it should be possible to generate variants of these Java methods that use more efficient representations and operations, e.g. the Java int values and integer operations when there is provably no wrap-around, in particular leveraging ACL2's the forms and the associated guard verification, which similarly help the Lisp compiler. Given that generating these shallowly embedded representations is inevitably more complicated and thus error-prone, the slower but safer interpreted evaluation could be still available as an option, at least in the absence of verification.

The Java code generated by ATJ can be called by external Java code, but not vice versa. Allowing the other call direction may involve suitable ACL2 stubs that correspond to the external code to be called.

The implementation of ATJ could be simplified by directly generating a Java abstract syntax and using a separable pretty printer to write abstract syntax to the file.

More ambitious projects are to (i) verify the correctness of AIJ's evaluator and primitive function implementations, and (ii) extend ATJ to generate a proof of correctness of the generated Java code, like a verifying compiler. Optimizing AIJ and generating shallowly embedded representations in ATJ make these verification tasks harder; an idea worth exploring is to perform a compositional verification of optimized Java code against unoptimized Java code and of the latter against ACL2 code.

The approach to generate Java code described in this paper, including the envisioned extensions described in this section, could be used to generate code in other programming languages. In particular, the UML class diagram in Figure 1 could be used for other object-oriented programming languages.

\section{Related Work}

The author is not aware of any Java or other code generator for ACL2.

Several theorem provers (Isabelle, PVS, Coq, etc.) include facilities to generate code in various programming languages (Standard ML, Ocaml, Haskell, Scala, C, Scheme, etc.) [8, 13, 7]. These code generators use shallow-embedding approaches, very different from ATJ and AIJ's deep-embedding approach. These code generators may be more relevant to future versions of ATJ and AIJ that use a shallow-embedding approach (see Section 6). However, the ACL2 language is quite different from the languages of those provers: first-order vs. higher-order, untyped with a fixed universe of (evaluation) values vs. typed with user-definable types, extra-logical guards vs. types that are part of the logic, and so on. Thus, only some of the ideas from those provers' code generators may be relevant to ACL2.

As discussed in Section 1, there are other ways for ACL2 code to interoperate with code in other programming languages, without the need for generating code in those programming languages from ACL2. However, this obviated need should be balanced against the issues with these approaches discussed in Section 1; different approaches may be best suited to different applications.

\section{Acknowledgements}

This work was supported by DARPA under Contract No. FA8750-15-C-0007. Thanks to Matt Kaufmann for useful discussions about the ACL2 evaluation semantics. Thanks to the anonymous reviewers for valuable suggestions. 


\section{References}

[1] Armed Bear Common Lisp (ABCL). https://abcl.org

[2] ACL2 Theorem Prover and Community Books: User Manual. http://www.cs.utexas.edu/ moore/ acl2/manuals/current/manual.

[3] APT (Automated Program Transformations). http://www.kestrel.edu/home/projects/apt

[4] CFFI: The Common Foreign Function Interface. https://common-lisp.net/project/cffi.

[5] Alessandro Coglio (2018): A Formalization of the ABNF Notation and a Verified Parser of ABNF Grammars. In: Proc. 10th Working Conference on Verified Software: Theories, Tools, and Experiments (VSTTE). To appear in Springer LNCS.

[6] Alessandro Coglio, Matt Kaufmann \& Eric Smith (2017): A Versatile, Sound Tool for Simplifying Definitions. In: Proc. 14th International Workshop on the ACL2 Theorem Prover and Its Applications (ACL2-2017), pp. 61-77, doi:10.4204/EPTCS.249.5

[7] Coq 8.8.1 Reference Manual. https://coq.inria.fr.

[8] Florian Haftmann with contributions from Lukas Bulwahn (2017): Code generation from Isabelle/HOL theories. https://isabelle.in.tum.de. Tutorial distributed with Isabelle/HOL.

[9] Java Native Interface JNI Specification. https://docs.oracle.com/javase/10/docs/specs/jni.

[10] Neil D. Jones, Carsten K. Gomard \& Peter Sestoft (1999): Partial Evaluation and Automatic Program Generation. Prentice Hall. http://www.itu.dk/people/sestoft/pebook.

[11] Matt Kaufmann \& J Strother Moore (1998): A Precise Description of the ACL2 Logic. Technical Report, Department of Computer Sciences, University of Texas at Austin. http://www.cs.utexas.edu/users/ moore/publications/km97a.pdf

[12] John McCarthy (1960): Recursive Functions of Symbolic Expressions and Their Computation by Machine, Part I. Communications of the ACM 3(4), pp. 184-195, doi:10.1145/367177.367199

[13] Nararajan Shankar (2017): A Brief Introduction to the PVS2C Code Generator. In: Proc. Workshop on Automated Formal Methods (AFM'17).

[14] Guy L. Steele (1990): Common Lisp the Language. Digital Press. https://www.cs.cmu.edu/Groups/ AI/html/cltl/cltl2.html. 